\title{
Explicit factorization of external coordinates in constrained Statistical Mechanics models
}

\author{
Pablo Echenique ${ }^{1,2 *}$ and Iván Calvo ${ }^{1,2}$ \\ 1 Departamento de Física Teórica, Facultad de Ciencias, Universidad de Zaragoza, \\ Pedro Cerbuna 12, 50009, Zaragoza, Spain. \\ 2 Instituto de Biocomputación y Física de los Sistemas Complejos (BIFI), \\ Edificio Cervantes, Corona de Aragón 42, 50009, Zaragoza, Spain.
}

October 25, 2018

\begin{abstract}
If a macromolecule is described by curvilinear coordinates or rigid constraints are imposed, the equilibrium probability density that must be sampled in Monte Carlo simulations includes the determinants of different mass-metric tensors. In this work, we explicitly write the determinant of the mass-metric tensor $G$ and of the reduced mass-metric tensor $g$, for any molecule, general internal coordinates and arbitrary constraints, as a product of two functions; one depending only on the external coordinates that describe the overall translation and rotation of the system, and the other only on the internal coordinates. This work extends previous results in the literature, proving with full generality that one may integrate out the external coordinates and perform Monte Carlo simulations in the internal conformational space of macromolecules. In addition, we give a general mathematical argument showing that the factorization is a consequence of the symmetries of the metric tensors involved. Finally, the determinant of the mass-metric tensor $G$ is computed explicitly in a set of curvilinear coordinates specially well-suited for general branched molecules.
\end{abstract}

PACS: 05.20.-y, 36.10.-k, 87.14.-g, 87.15.-v, 87.15.Aa, 89.75.-k

\section{Introduction}

Monte Carlo simulations are among the most useful tools for studying the behavior of macromolecules in thermal equilibrium [1-8]. Typically, the simulations are carried out in the coordinate space, i.e., the momenta are averaged out and Monte Carlo movements that only change the coordinates of the system are designed.

Also, the most interesting properties of macromolecules depend only on conformational transitions in the internal subspace of the whole coordinate space. The protein folding problem [9-12], the docking of ligands to proteins [13], or proteins to proteins [14], the prediction of Raman [15,16], IR [17,18], CD [19], VCD [20,21], NMR [22,23] spectra, etc. are tasks that require knowledge of the probability density in the conformational space only, i.e., having averaged out the external coordinates that describe overall translations and rotations of the system.

*Corresponding author. E-mail address: pnique@unizar.es 
If Cartesian coordinates are used, the integration over the momenta produces a constant factor (which depends on the temperature $T$ but does not depend on the coordinates) and the marginal probability density in the coordinate space resembles the common Boltzmann weight but using the potential energy $V\left(x^{\mu}\right)$ instead of the whole energy:

$$
p_{c}\left(x^{\mu}\right)=\frac{\exp \left[-\beta V\left(x^{\mu}\right)\right]}{\int \mathrm{d} x^{\nu} \exp \left[-\beta V\left(x^{\nu}\right)\right]} .
$$

Typically the potential energy does not change under global translations and rotations of the system. In addition, as we have already mentioned, one is normally not interested in averages of observables that depend on these degrees of freedom. Hence, it would be convenient to average them out from eq. (1.1). However, this cannot be done in Cartesian coordinates: one must use a set of coordinates adapted to overall translations and rotations.

In the simulation of macromolecules, it is customary $[8,24-30]$ to define a set of curvilinear coordinates $q^{\mu}$ in which the first six ones, denoted by $q^{A}$, are called external coordinates and parametrize the system overall position, specifying the position of a selected point (normally an atom), and rotation, via three Euler angles (see sec. 21). The remaining $3 n-6$ coordinates (where $n$ is the number of mass points or atoms) are called internal coordinates and will be denoted herein by $q^{a}$.

This change of coordinates modifies the mass-metric tensor in the kinetic energy. Thus, when the momenta are averaged out and the marginal probability density in the whole coordinate space is considered, the square root of the determinant of the mass-metric tensor (which now does depend on the coordinates) shows up:

$$
p_{w}\left(q^{\mu}\right)=\frac{\operatorname{det}^{\frac{1}{2}} G\left(q^{A}, q^{a}\right) \exp \left[-\beta V\left(q^{a}\right)\right]}{\int \mathrm{d} q^{B} \mathrm{~d} q^{b} \operatorname{det}^{\frac{1}{2}} G\left(q^{B}, q^{b}\right) \exp \left[-\beta V\left(q^{b}\right)\right]} .
$$

More interestingly, if holonomic constraints are imposed on the system (the so-called classical rigid model [31-33]), the reduced mass-metric tensor on the constrained hypersurface appears in the kinetic energy. Hence, when the momenta are integrated out from the joint probability density in the phase space, the square root of its determinant occurs:

$$
p_{r}\left(q^{u}\right)=\frac{\operatorname{det}^{\frac{1}{2}} g\left(q^{A}, q^{i}\right) \exp \left[-\beta V_{\Sigma}\left(q^{i}\right)\right]}{\int \mathrm{d} q^{B} \mathrm{~d} q^{j} \operatorname{det}^{\frac{1}{2}} g\left(q^{B}, q^{j}\right) \exp \left[-\beta V_{\Sigma}\left(q^{j}\right)\right]},
$$

where $V_{\Sigma}$ stands for the potential energy in the constrained hypersurface $\Sigma, q^{u} \equiv$ $\left(q^{A}, q^{i}\right)$ denotes the soft coordinates, among which the external ones $q^{A}$ are included, and $q^{i}$ denotes the soft internal coordinates.

If, on the other hand, the constraints are imposed via a steep potential that energetically penalizes the conformations that leave the constrained hypersurface (the so-called classical stiff model [30-33]), the probability density is the same as in eq. (1.2) except for the determinant of the Hessian matrix $\mathcal{H}$ of the constraining potential that appears when the hard coordinates are averaged out and for the fact that all the functions are evaluated on the constrained hypersurface and, consequently, depend only on the soft coordinates $q^{u}$ :

$$
p_{s}\left(q^{u}\right)=\frac{\operatorname{det}^{\frac{1}{2}} G\left(q^{A}, q^{i}\right) \operatorname{det}^{-\frac{1}{2}} \mathcal{H}\left(q^{i}\right) \exp \left[-\beta V_{\Sigma}\left(q^{i}\right)\right]}{\int \mathrm{d} q^{B} \mathrm{~d} q^{j} \operatorname{det}^{\frac{1}{2}} G\left(q^{B}, q^{j}\right) \operatorname{det}^{-\frac{1}{2}} \mathcal{H}\left(q^{j}\right) \exp \left[-\beta V_{\Sigma}\left(q^{j}\right)\right]} .
$$


Finally, the Fixman's compensating potential [31-34], denoted by $V_{F}$ and which is customarily used to reproduce the stiff equilibrium distribution using rigid molecular dynamics simulations $[8,31,35]$, is also expressed as a function of these determinants:

$$
V_{F}\left(q^{u}\right):=\frac{R T}{2} \ln \left[\frac{\operatorname{det}^{\frac{1}{2}} g\left(q^{A}, q^{i}\right) \operatorname{det}^{\frac{1}{2}} \mathcal{H}\left(q^{i}\right)}{\operatorname{det}^{\frac{1}{2}} G\left(q^{A}, q^{i}\right)}\right] .
$$

One should note that the fields of application of the different mass-metric tensor determinants are distinct. The Fixman's compensating potential above is only meant to allow that the stiff distribution in eq. (1.4) be sampled in rigid Molecular Dynamics simulations [33,35-45]. and it should never be included in Monte Carlo simulations. On the other hand, if one chooses as his physical description the whole space, the rigid or the stiff model, the probability densities that must be sampled are the ones in eqs. (1.2), (1.3) and (1.4), respectively $[30-34,44,46]$. Due to the averaging out of the momenta in these expressions, the determinants of either $G$ or $g$ show up, hence, if Monte Carlo simulations of these models are to be performed, these corrections (which are related to but different from the Fixman's compensating potential) should be included or, otherwise, shown to be negligible. The discussion found in the literature about the necessity of including these terms [47] is based on different simplifications, approximations and misconceptions. On one side, since Gō and Scheraga showed some decades ago that the determinant of $G$, for a serial polymer with constant bond angles and bond lengths, does not depend on the conformation of the molecule [30], it is customarily neglected in the literaure. However, this must be understood as an approximation, since, as some authors have recognized $[30,39,48,49]$, the constrained values of the hard coordinates depend on the soft ones even in the case of simple force-fields, due to the long-range energy terms and, in such a case, $\operatorname{det} G$ does depend on the $q^{i}$, rendering pertintent its inclusion in the basic equations. Moreover, the determinant of the Hessian $\mathcal{H}$ of the constraining part of the potential is also assumed to be independent of conformation by most authors [30,33,50]. This is also an approximation, due to the same reasons presented above, and should be assessed in each case, however, if these two approximations are made (the neglect of $\operatorname{det} G$ and of $\operatorname{det} \mathcal{H}$ ), the Fixman's compensating potential in eq. (1.5) depends only on $\operatorname{det} g$, just as the correcting term in the rigid case. This is one of the sources of confusion and the reason that the relevance of mass-metric effects is associated only with the conformational dependence of the determinant of the reduced mass-metric tensor $g$ [47]. One should also note that most authors accept that the correct constrained model is the stiff one $[33,34,38,42,47,50,51]$, and, therefore, no works are written in which the rigid probability density in eq. (1.3) is sampled. In our opinion, the question whether the rigid or the stiff model should be used to approximate the real quantum mechanical statistics of an arbitrary organic molecule has not been satisfactorily answered yet. For discussions about the topic, see references $[30,32,42,42,44,46,49,52]$. In this work, we adopt the cautious position that any of the two models may be useful in certain cases or for certain purposes and we study them both on equal footing. All these approximations together are the reason of the fact that no determinants are included in Monte Carlo simulations and that the Fixman's compensating potential is erroneusly regarded as playing a role outside Molecular Dynamics simulations.

In the three physical models, given by eqs. (1.2), (1.3) and (1.4), and in the Fixman's potential written above, neither the potential energy nor the Hessian matrix of the constraining potential depend on the external coordinates. Therefore, it would be very convenient to integrate them out in order to obtain a simpler probability density depending only on the internal coordinates. Such an improvement may render the coding of computer applications and the visualization of molecules easier, since all the movements in the molecule may be performed fixing an atom in space and keeping the orientation 
of the system with respect to a set of axes fixed in space constant [53]. Moreover, the insight gained and the usefulness of the calculations herein in further analytical studies constitute additional benefits. Regarding Monte Carlo simulations, it is not clear that much computational effort will be saved for macromolecules, since the gain expected when reducing the degrees of freedom from $M+6$ to $M$ (where $M$ is the number of soft internal coordinates) is only appreciable if $M$ is small and the difficulties arising from the use of curvilinear coordinates may well be more important. However, in cases where the use of curvilinear coordinates is a must, such as the simulation of constrained systems, the calculations in this work allow to save a time (which will depend on the size of the system) that, otherwise, would be wasted in movements of the external coordinates. Finally, if the molecule treated is small, as it is common in ab initio Quantum Mechanical calculations in model peptides [54-58], the relevance of omitting the externals may be considerable. In a work recently done in our group [31], in which the model dipeptide HCO-L-Ala- $\mathrm{NH}_{2}$ is studied, the soft internals are two: the Ramachandran angles $\phi$ and $\psi$; hence, $M=2$ and the formulae in this work have permitted to reduce the number of degrees of freedom from 8 to 2 . This situation is very common in the literature [54-58].

Now, for the resulting expressions to be manageable, the determinant of the massmetric tensor $G$, in $p_{w}$ or $p_{s}$, and the determinant of $g$, in the rigid case, should factorize as a product of a function that depends only on the external coordinates and another function that depends only on the internal ones. Then the function depending on the external coordinates, could be integrated out in the probability densities $p_{w}, p_{r}$ and $p_{s}$ or taken out of the logarithm in $V_{F} 1$.

For some simple examples, it has already been proven in the literature that this factorization actually happens. In ref. 30, the determinant of $G$ is shown to factorize for a serial polymer in a particular set of curvilinear coordinates. In ref. 47, the determinant of $g$ is shown to factorize for the same system, in similar coordinates, with frozen bond lengths and bond angles.

In this work, we generalize these results, showing that they hold in arbitrary internal coordinates (for general branched molecules) and with arbitrary constraints. Perhaps more importantly, we provide explicit expression for the functions involved in the factorization. It is worth remarking that, although the calculations herein have been performed thinking in macromolecules as target system, they are completely general and applicable to any classical system composed by discrete mass points.

In sec. 2. we present the notation and conventions that will be used throughout the article. In sec. 3, we explicitly factorize the determinant of the reduced mass-metric tensor $g$ as a product of a function that depends only on the external coordinates and another function that depends on arbitrary internal coordinates; no specific form is assumed for the constraints. In sec. 4 , we perform the analogous calculations for the determinant of the mass-metric tensor $G$ and sec. 5 is devoted to the conclusions. In appendix A, the general mathematical argument underlying these results is given. Finally, in appendix B, the determinant of the mass-metric tensor $G$ is computed in the set of curvilinear coordinates introduced in ref. 24, which turn out to be convenient for dealing with general branched molecules. Moreover, we show that the classical formula for serial polymers [30] is actually valid for any macromolecule.

\section{General set-up and definitions}

The system under scrutiny will be a set of $n$ mass points termed atoms. This section is devoted to introduce certain notational conventions that will be used extensively in the

\footnotetext{
${ }^{1}$ What really happens, (see secs. 3 and 4 is that the factor that depends on the external coordinates is the same for $\operatorname{det} G$ and $\operatorname{det} g$. Hence, it divides out in eq. 1.5) (see sec. 5).
} 
rest of the paper.

- The superindex $T$ indicates matrix transposition. By $\vec{a}^{T}$ we shall understand the row vector $\left(a^{1}, a^{2}, a^{3}\right)$.

- The Cartesian coordinates of the atom $\alpha$ in a set of axes fixed in space are denoted by $\vec{x}_{\alpha}$. The subscript $\alpha$ runs from 1 to $n$.

- The curvilinear coordinates suitable to integrate out the external degrees of freedom will be denoted by $q^{\mu}, \mu=1, \ldots, 3 n$. We shall often use $N:=3 n$ for the total number of degrees of freedom.

- We choose the coordinates $q^{\mu}$ so that the first six are external coordinates. They are denoted by $q^{A}$ and their ordering is $q^{A} \equiv(X, Y, Z, \phi, \theta, \psi)$. The first three ones, $\vec{X}^{T}:=(X, Y, Z)$, describe the overall position of the system. The three angles $(\phi, \theta, \psi)$ are related to its overall orientation. More concretely, they give the orientation of a frame fixed in the system with respect to the frame fixed in space.

- To define the set of axes fixed in the system, we select three atoms (denoted by 1,2 and 3 ) in such a way that $\vec{X}$ is the position of atom 1 (i.e., $\vec{x}_{1}=\vec{X}$ ). The orientation of the fixed axes $\left(x^{\prime}, y^{\prime}, z^{\prime}\right)$ is chosen such that atom 2 lies in the positive half of the $z^{\prime}$-axis and atom 3 is contained in the $\left(x^{\prime}, z^{\prime}\right)$-plane, in the positive half of the $x^{\prime}$-axis (see fig. 11). The position of atom $\alpha$ in these axes is denoted by $\vec{x}_{\alpha}^{\prime}$.

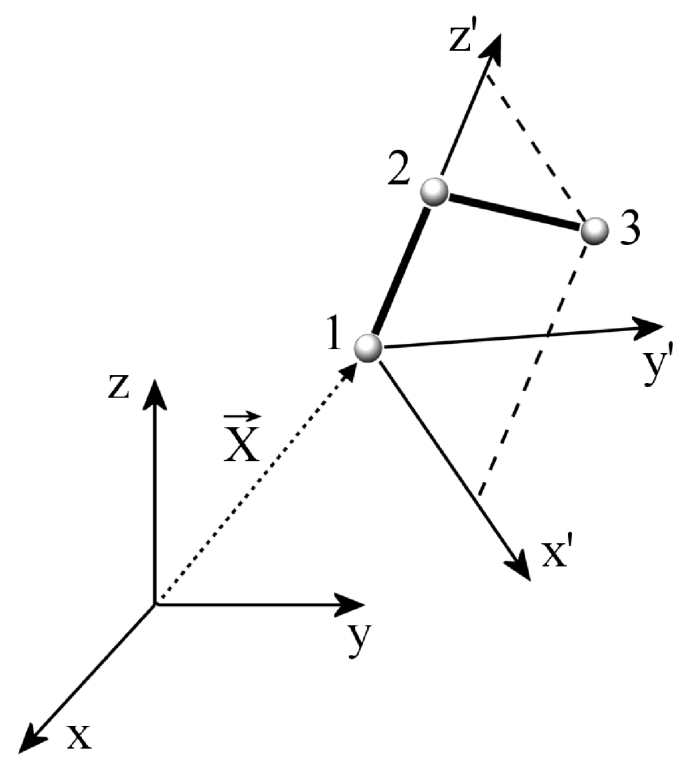

Figure 1: Definition of the axes fixed in the system.

- Let $E(\phi, \theta, \psi)$ be the Euler rotation matrix (in the ZYZ convention) that takes a vector of primed components $\vec{a}^{\prime}$ to the frame fixed in space, i.e., $\vec{a}=E(\phi, \theta, \psi) \vec{a}^{\prime}$. Its explicit expression is the following:

$$
E(\phi, \theta, \psi)=\underbrace{\left(\begin{array}{crr}
\cos \phi & -\sin \phi & 0 \\
\sin \phi & \cos \phi & 0 \\
0 & 0 & 1
\end{array}\right)}_{\Phi(\phi)} \underbrace{\left(\begin{array}{ccc}
-\cos \theta & 0 & \sin \theta \\
0 & 0 \\
-\sin \theta & 0 & -\cos \theta
\end{array}\right)}_{\Theta(\theta)} \underbrace{\left(\begin{array}{ccc}
\cos \psi & -\sin \psi & 0 \\
\sin \psi & \cos \psi & 0 \\
0 & 0 & 1
\end{array}\right)}_{\Psi(\psi)} .
$$


The unusual minus signs of the cosines in the diagonal of matrix $\Theta(\theta)$ come from the fact that, due to frequent biochemical conventions, the rotation with respect to the $y$-axis is of angle $\tilde{\theta}:=\pi-\theta$.

- The coordinates $q^{\mu}$ are split into $\left(q^{A}, q^{a}\right), a=7, \ldots, N$. The coordinates $q^{a}$ are said internal coordinates and determine the positions of the atoms in the frame fixed in the system. The transformation from the Cartesian coordinates $\vec{x}_{\alpha}$ to the curvilinear coordinates $q^{\mu}$ may be written as follows:

$$
\vec{x}_{\alpha}=\vec{X}+E(\phi, \theta, \psi) \vec{x}_{\alpha}^{\prime}\left(q^{a}\right) \quad \alpha=1, \ldots, n .
$$

- The coordinates $q^{a}$ parameterize what we shall call the internal subspace, denoted by $\mathcal{I}$. Assume that $L$ independent constraints are imposed on $\mathcal{I}$, so that only points on a hypersurface $\Sigma \subset \mathcal{I}$ of dimension $M:=N-L-6$ are allowed. Then, we choose a splitting $q^{a} \equiv\left(q^{i}, q^{I}\right)$, with $i=7, \ldots, M+6$ and $I=M+7, \ldots, N$, where $q^{i}$ (the internal soft coordinates) parameterize $\Sigma$, and $q^{I}$ (the hard coordinates) are functions of the soft coordinates:

$$
q^{I}=f^{I}\left(q^{i}\right) \quad I=M+7, \ldots, N .
$$

If these constraints are used, together with eq. (2.2), the Cartesian position of any atom may be parameterized with the set of all soft coordinates, denoted by $q^{u} \equiv\left(q^{A}, q^{i}\right)$, with $u=1, \ldots, M+6$, as follows:

$$
\vec{x}_{\alpha}=\vec{X}+E(\phi, \theta, \psi) \vec{x}_{\alpha}^{\prime}\left(q^{i}, f^{I}\left(q^{i}\right)\right) \quad \alpha=1, \ldots, n .
$$

- In table 1, a summary of the indices used is given.

\begin{tabular}{llll} 
Indices & Range & Number & Description \\
\hline$\alpha, \beta, \gamma, \ldots$ & $1, \ldots, n$ & $n$ & Atoms \\
$p, q, r, s, \ldots$ & $1,2,3$ & 3 & Components of trivectors \\
$\mu, \nu, \rho, \ldots$ & $1, \ldots, N$ & $N=3 n$ & All coordinates \\
$A, B, C, \ldots$ & $1, \ldots, 6$ & 6 & External coordinates \\
$a, b, c, \ldots$ & $7, \ldots, N$ & $N-6$ & Internal coordinates \\
$i, j, k, \ldots$ & $7, \ldots, M+6$ & $M$ & Soft internal coordinates \\
$I, J, K, \ldots$ & $M+7, \ldots, N$ & $L=N-M-6$ & Hard internal coordinates \\
$u, v, w, \ldots$ & $1, \ldots, M+6$ & $M+6$ & All soft coordinates
\end{tabular}

Table 1: Definition of the indices used.

\section{Constrained case}

The reduced mass-metric tensor, in the constrained hypersurface $\Sigma$ plus the external subspace spanned by the $q^{A}$, may be written as follows:

$$
g_{v w}\left(q^{u}\right):=\sum_{\mu=1}^{N} \frac{\partial x^{\mu}\left(q^{u}\right)}{\partial q^{v}} m_{\mu} \frac{\partial x^{\mu}\left(q^{u}\right)}{\partial q^{w}} .
$$

In matrix notation, this is written as 


$$
g=J_{c}^{T} M J_{c},
$$

where $c$ stands for constrained and $M$ is the diagonal $N \times N$ mass matrix given by

$$
M:=\left(\begin{array}{ccc}
m_{1}^{(3)} & & 0 \\
& \ddots & \\
0 & & m_{n}^{(3)}
\end{array}\right), \text { with } m_{\alpha}^{(3)}:=m_{\alpha} \underbrace{\left(\begin{array}{ccc}
1 & 0 & 0 \\
0 & 1 & 0 \\
0 & 0 & 1
\end{array}\right)}_{I^{(3)}} .
$$

Using eq. (2.4) and noting that the derivatives with respect to the externals, $q^{A}$, only affect the $\vec{X}$ vector and the Euler rotation matrix $E$, while differentiation with respect to soft internals, $q^{i}$, only act on the $\vec{x}_{\alpha}^{\prime}$, we have that $J_{c}$ is the $N \times(M+6)$ matrix

$$
J_{c}=\left(\begin{array}{cccc|cc}
I^{(3)} & & 0 & & \\
I^{(3)} & \frac{\partial E}{\partial \phi} \vec{x}_{2}^{\prime} & \frac{\partial E}{\partial \theta} \vec{x}_{2}^{\prime} & \frac{\partial E}{\partial \psi} \vec{x}_{2}^{\prime} & \cdots & E \frac{\partial \vec{x}_{2}^{\prime}}{\partial q^{j}} \cdots \\
\vdots & \vdots & \vdots & \vdots & & \vdots \\
I^{(3)} & \frac{\partial E}{\partial \phi} \vec{x}_{\alpha}^{\prime} & \frac{\partial E}{\partial \theta} \vec{x}_{\alpha}^{\prime} & \frac{\partial E}{\partial \psi} \vec{x}_{\alpha}^{\prime} & \cdots & E \frac{\partial \vec{x}_{\alpha}^{\prime}}{\partial q^{j}} \cdots \\
\vdots & \vdots & \vdots & \vdots & & \vdots
\end{array}\right)
$$

If we perform the matrix multiplications in eq. (3.2), we obtain

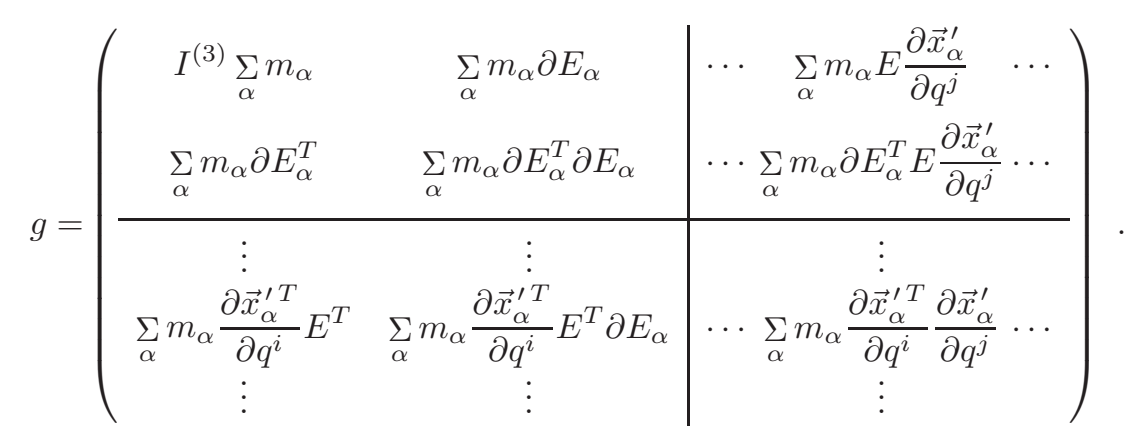

All the sums in $\alpha$ can be understood as ranging from 1 to $n$ if we note that $\vec{x}_{1}^{\prime}=\overrightarrow{0}$. In the bottom right block, the fact that $E$ is an orthogonal matrix (i.e., that $E^{T} E=I^{(3)}$ ) has been used, and we have defined the $3 \times 3$ block as

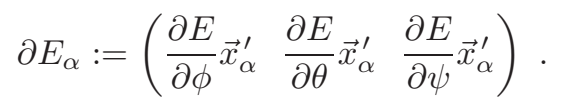

We can write $g$ as

$$
g:=\left(\begin{array}{cc}
E & 0 \\
0 & I^{(M+3)}
\end{array}\right) g_{1}\left(\begin{array}{cc}
E^{T} & 0 \\
0 & I^{(M+3)}
\end{array}\right),
$$

where $I^{(M+3)}$ is the $(M+3) \times(M+3)$ identity matrix and $g_{1}$ is defined as 


$$
g_{1}:=\left(\begin{array}{cc|ccc}
I^{(3)} \sum_{\alpha} m_{\alpha} & \sum_{\alpha} m_{\alpha} E^{T} \partial E_{\alpha} & \cdots & \sum_{\alpha} m_{\alpha} \frac{\partial \vec{x}_{\alpha}^{\prime}}{\partial q^{j}} & \cdots \\
\sum_{\alpha} m_{\alpha} \partial E_{\alpha}^{T} E & \sum_{\alpha} m_{\alpha} \partial E_{\alpha}^{T} E E^{T} \partial E_{\alpha} & \cdots & \sum_{\alpha} m_{\alpha} \partial E_{\alpha}^{T} E \frac{\partial \vec{x}_{\alpha}^{\prime}}{\partial q^{j}} \cdots \\
\hline \vdots & \vdots & & \vdots \\
\sum_{\alpha} m_{\alpha} \frac{\partial \vec{x}_{\alpha}^{\prime T}}{\partial q^{i}} & \sum_{\alpha} m_{\alpha} \frac{\partial \vec{x}_{\alpha}^{\prime T}}{\partial q^{i}} E^{T} \partial E_{\alpha} & \cdots & \sum_{\alpha} m_{\alpha} \frac{\partial \vec{x}_{\alpha}^{\prime T}}{\partial q^{i}} \frac{\partial \vec{x}_{\alpha}^{\prime}}{\partial q^{j}} & \cdots \\
\vdots & \vdots & & \vdots
\end{array}\right) .
$$

Note that $I^{(3)}=E E^{T}$ has been introduced in the bottom right $3 \times 3$ submatrix of the top left block.

Next, we introduce some simplifying notation for the matrices $E^{T} \partial E_{\alpha}$ :

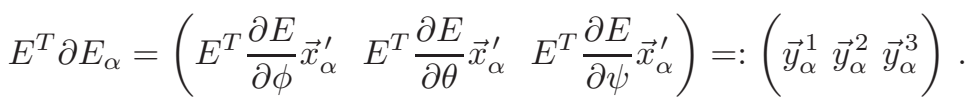

Defining

$$
g_{1}^{\alpha}:=\left(\begin{array}{cccc|ccc}
I^{(3)} & \vec{y}_{\alpha}^{1} & \vec{y}_{\alpha}^{2} & \vec{y}_{\alpha}^{3} & \cdots & \frac{\partial \vec{x}_{\alpha}^{\prime}}{\partial q^{j}} & \cdots \\
\vec{y}_{\alpha}^{1 T} & \vec{y}_{\alpha}^{1 T} \vec{y}_{\alpha}^{1} & \vec{y}_{\alpha}^{1 T} \vec{y}_{\alpha}^{2} & \vec{y}_{\alpha}^{1 T} \vec{y}_{\alpha}^{3} & \cdots & \vec{y}_{\alpha}^{1 T} \frac{\partial \vec{x}_{\alpha}^{\prime}}{\partial q^{j}} & \cdots \\
\vec{y}_{\alpha}^{2 T} & \vec{y}_{\alpha}^{2 T} \vec{y}_{\alpha}^{1} & \vec{y}_{\alpha}^{2 T} \vec{y}_{\alpha}^{2} & \vec{y}_{\alpha}^{2 T} \vec{y}_{\alpha}^{3} & \cdots & \vec{y}_{\alpha}^{2 T} \frac{\partial \vec{x}_{\alpha}^{\prime}}{\partial q^{j}} & \cdots \\
\vec{y}_{\alpha}^{3 T} & \vec{y}_{\alpha}^{3 T} \vec{y}_{\alpha}^{1} & \vec{y}_{\alpha}^{3 T} \vec{y}_{\alpha}^{2} & \vec{y}_{\alpha}^{3 T} \vec{y}_{\alpha}^{3} & \cdots & \vec{y}_{\alpha}^{3 T} \frac{\partial \vec{x}_{\alpha}^{\prime}}{\partial q^{j}} & \cdots \\
\hline \vdots & \vdots & \vdots & \vdots & & \vdots \\
\frac{\partial \vec{x}_{\alpha}^{\prime T}}{\partial q^{i}} & \frac{\partial \vec{x}_{\alpha}^{\prime T}}{\partial q^{i}} \vec{y}_{\alpha}^{1} & \frac{\partial \vec{x}_{\alpha}^{\prime T}}{\partial q^{i}} \vec{y}_{\alpha}^{2} & \frac{\partial \vec{x}_{\alpha}^{\prime T}}{\partial q^{i}} \vec{y}_{\alpha}^{3} & \cdots \frac{\partial \vec{x}_{\alpha}^{\prime T}}{\partial q^{i}} \frac{\partial \vec{x}_{\alpha}^{\prime}}{\partial q^{j}} & \cdots \\
\vdots & \vdots & \vdots & \vdots & & \vdots
\end{array}\right),
$$

we have that

$$
g_{1}=\sum_{\alpha} m_{\alpha} g_{1}^{\alpha}
$$

Now, the vectors $\vec{y}_{\alpha}^{p}$ may be extracted from $g_{1}^{\alpha}$ as follows:

$$
g_{1}^{\alpha}=Y_{\alpha}^{T}\left(\begin{array}{cc|ccc}
I^{(3)} & I^{(3)} & \cdots & \frac{\partial \vec{x}_{\alpha}^{\prime}}{\partial q^{j}} & \cdots \\
I^{(3)} & I^{(3)} & \cdots & \frac{\partial \vec{x}_{\alpha}^{\prime}}{\partial q^{j}} & \cdots \\
\hline \vdots & \vdots & \vdots \\
\frac{\partial \vec{x}_{\alpha}^{\prime T}}{\partial q^{i}} & \frac{\partial \vec{x}_{\alpha}^{\prime T}}{\partial q^{i}} & \cdots \frac{\partial \vec{x}_{\alpha}^{\prime T}}{\partial q^{i}} \frac{\partial \vec{x}_{\alpha}^{\prime}}{\partial q^{j}} \cdots \\
\vdots & \vdots & \vdots
\end{array}\right) Y_{\alpha}
$$


where

$$
Y_{\alpha}:=\left(\begin{array}{ccc}
I^{(3)} & 0 & 0 \\
0 & \vec{y}_{\alpha}^{1} \vec{y}_{\alpha}^{2} \vec{y}_{\alpha}^{3} & 0 \\
0 & 0 & I^{(M)}
\end{array}\right)
$$

and the central matrix in eq. (3.12) only depends on the soft internal coordinates. After some lengthy calculations, one shows that

$$
\left(\begin{array}{lll}
\vec{y}_{\alpha}^{1} & \vec{y}_{\alpha}^{2} & \vec{y}_{\alpha}^{3}
\end{array}\right)=\underbrace{\left(\begin{array}{ccc}
0 & -x_{\alpha}^{\prime 3} & x_{\alpha}^{\prime 2} \\
x_{\alpha}^{\prime 3} & 0 & -x_{\alpha}^{\prime 1} \\
-x_{\alpha}^{\prime 2} & x_{\alpha}^{\prime 1} & 0
\end{array}\right)}_{v\left(\vec{x}_{\alpha}^{\prime}\right)} \underbrace{\left(\begin{array}{ccc}
\sin \theta \cos \psi & \sin \psi & 0 \\
-\sin \theta \sin \psi & \cos \psi & 0 \\
\cos \theta & 0 & -1
\end{array}\right)}_{W(\theta, \psi)}
$$

Thus, the matrix $Y_{\alpha}$ in eq. (3.13) may be written as

$$
Y_{\alpha}=\left(\begin{array}{ccc}
I^{(3)} & 0 & 0 \\
0 & v\left(\vec{x}_{\alpha}^{\prime}\right) & 0 \\
0 & 0 & I^{(M)}
\end{array}\right)\left(\begin{array}{ccc}
I^{(3)} & 0 & 0 \\
0 & W(\theta, \psi) & 0 \\
0 & 0 & I^{(M)}
\end{array}\right) .
$$

If we take this expression to eq. (3.12) and use that, for any pair of vectors $\vec{a}$ and $\vec{b}$, $\vec{a}^{T} v(\vec{b})=(\vec{a} \times \vec{b})^{T}$ and $v^{T}(\vec{b}) \vec{a}=\vec{a} \times \vec{b}$, where $\times$ denotes the usual vector cross product, we may rewrite eq. (3.12) as follows:

$$
g_{1}^{\alpha}=\left(\begin{array}{ccc}
I^{(3)} & 0 & 0 \\
0 & W^{T}(\theta, \psi) & 0 \\
0 & 0 & I^{(M)}
\end{array}\right) g_{2}^{\alpha}\left(\begin{array}{ccc}
I^{(3)} & 0 & 0 \\
0 & W(\theta, \psi) & 0 \\
0 & 0 & I^{(M)}
\end{array}\right),
$$

where $g_{2}^{\alpha}$ is defined as

$$
g_{2}^{\alpha}=\left(\begin{array}{cc|ccc}
I^{(3)} & v\left(\vec{x}_{\alpha}^{\prime}\right) & \cdots & \frac{\partial \vec{x}_{\alpha}^{\prime}}{\partial q^{j}} & \cdots \\
v^{T}\left(\vec{x}_{\alpha}^{\prime}\right) & v^{T}\left(\vec{x}_{\alpha}^{\prime}\right) v\left(\vec{x}_{\alpha}^{\prime}\right) & \cdots \frac{\partial \vec{x}_{\alpha}^{\prime}}{\partial q^{j}} \times \vec{x}_{\alpha}^{\prime} & \cdots \\
\hline \vdots & \vdots & \vdots \\
\frac{\partial \vec{x}_{\alpha}^{\prime T}}{\partial q^{i}} & \left(\frac{\partial \vec{x}_{\alpha}^{\prime}}{\partial q^{i}} \times \vec{x}_{\alpha}^{\prime}\right)^{T} & \cdots \frac{\partial \vec{x}_{\alpha}^{\prime T}}{\partial q^{i}} \frac{\partial \vec{x}_{\alpha}^{\prime}}{\partial q^{j}} & \cdots \\
\vdots & \vdots & \vdots
\end{array}\right) .
$$

At this point, we insert eq. (3.16) in eq. (3.11) and take the $W$ matrices out of the sum. Since $\operatorname{det} W(\theta, \psi)=\operatorname{det} W^{T}(\theta, \psi)=-\sin \theta$, we obtain

$$
\operatorname{det} g_{1}=\sin ^{2} \theta \operatorname{det} \underbrace{\left(\sum_{\alpha} m_{\alpha} g_{2}^{\alpha}\right)}_{g_{2}} \text {. }
$$

Recalling that $\operatorname{det} E=\operatorname{det} E^{T}=1$, from eq. (3.7), we have that

$$
\operatorname{det} g\left(q^{A}, q^{i}\right)=\operatorname{det} g_{1}\left(q^{A}, q^{i}\right)=\sin ^{2} \theta \operatorname{det} g_{2}\left(q^{i}\right),
$$

and the factorization of the external coordinates has been finally accomplished, since $g_{2}$ is the following matrix, which depends only on the soft internal coordinates $q^{i}$ : 


$$
g_{2}=\left(\begin{array}{cc|ccc}
m_{\text {tot }} I^{(3)} & m_{\text {tot }} v(\vec{R}) & \cdots & m_{t o t} \frac{\partial \vec{R}}{\partial q^{j}} & \cdots \\
m_{t o t} v^{T}(\vec{R}) & \mathcal{J} & \cdots \sum_{\alpha} m_{\alpha} \frac{\partial \vec{x}_{\alpha}^{\prime}}{\partial q^{j}} \times \vec{x}_{\alpha}^{\prime} \cdots \\
\hline \vdots & \vdots & \vdots \\
m_{t o t} \frac{\partial \vec{R}}{\partial q^{i}} & \sum_{\alpha} m_{\alpha}\left(\frac{\partial \vec{x}_{\alpha}^{\prime}}{\partial q^{i}} \times \vec{x}_{\alpha}^{\prime}\right)^{T} & \cdots \sum_{\alpha} m_{\alpha} \frac{\partial \vec{x}_{\alpha}^{\prime T}}{\partial q^{i}} \frac{\partial \vec{x}_{\alpha}^{\prime}}{\partial q^{j}} \cdots \\
\vdots & \vdots & \vdots
\end{array}\right),
$$

where we have defined the total mass of the system $m_{t o t}:=\sum_{\alpha} m_{\alpha}$, the position of the center of mass of the system in the primed reference frame $\vec{R}:=m_{t o t}^{-1} \sum_{\alpha} m_{\alpha} \vec{x}_{\alpha}^{\prime}$ and the inertia tensor of the system, also in the primed reference frame:

$$
\mathcal{J}:=\left(\begin{array}{ccc}
\sum_{\alpha} m_{\alpha}\left(\left(x_{\alpha}^{\prime 2}\right)^{2}+\left(x_{\alpha}^{\prime 3}\right)^{2}\right) & -\sum_{\alpha} m_{\alpha} x_{\alpha}^{\prime 1} x_{\alpha}^{\prime 2} & -\sum_{\alpha} m_{\alpha} x_{\alpha}^{\prime 1} x_{\alpha}^{\prime 3} \\
-\sum_{\alpha} m_{\alpha} x_{\alpha}^{\prime 1} x_{\alpha}^{\prime 2} & \sum_{\alpha} m_{\alpha}\left(\left(x_{\alpha}^{\prime 1}\right)^{2}+\left(x_{\alpha}^{\prime 3}\right)^{2}\right) & -\sum_{\alpha} m_{\alpha} x_{\alpha}^{\prime 2} x_{\alpha}^{\prime 3} \\
-\sum_{\alpha} m_{\alpha} x_{\alpha}^{\prime 1} x_{\alpha}^{\prime 3} & -\sum_{\alpha} m_{\alpha} x_{\alpha}^{\prime 2} x_{\alpha}^{\prime 3} & \sum_{\alpha} m_{\alpha}\left(\left(x_{\alpha}^{\prime \prime}\right)^{2}+\left(x_{\alpha}^{\prime 2}\right)^{2}\right)
\end{array}\right) .
$$

\section{Unconstrained case}

If no constraints are assumed and the system lives in the whole internal space $\mathcal{I}$ plus the external subspace spanned by the $q^{A}$, the Cartesian coordinates of the $n$ atoms must be expressed using eq. (2.2), instead of eq. (2.4).

We now wish to calculate the determinant of the whole-space mass-metric tensor in the coordinates $q^{\mu}$ :

$$
G_{\nu \rho}\left(q^{\mu}\right):=\sum_{\sigma=1}^{N} \frac{\partial x^{\sigma}\left(q^{\mu}\right)}{\partial q^{\nu}} m_{\sigma} \frac{\partial x^{\sigma}\left(q^{\mu}\right)}{\partial q^{\rho}}
$$

which, in matrix form, reads

$$
G=J^{T} M J .
$$

The only difference with eq. (3.2) is that, instead of the rectangular matrix $J_{c}$ (see eq. (3.4) ), in the above expression the full Jacobian matrix of the change of coordinates from Cartesian to curvilinear coordinates appears:

$$
J_{\rho}^{\sigma}\left(q^{\mu}\right):=\frac{\partial x^{\sigma}\left(q^{\mu}\right)}{\partial q^{\rho}} .
$$

Obviously, one can deduce the factorization of $\operatorname{det} G$ as a particular case of the results of sec. 3 with $L=0$, so that the indices $i, j$ now run over all internal coordinates $q^{a}$. Explicitly,

$$
\operatorname{det} G\left(q^{A}, q^{a}\right)=\sin ^{2} \theta \operatorname{det} G_{2}\left(q^{a}\right),
$$

with 


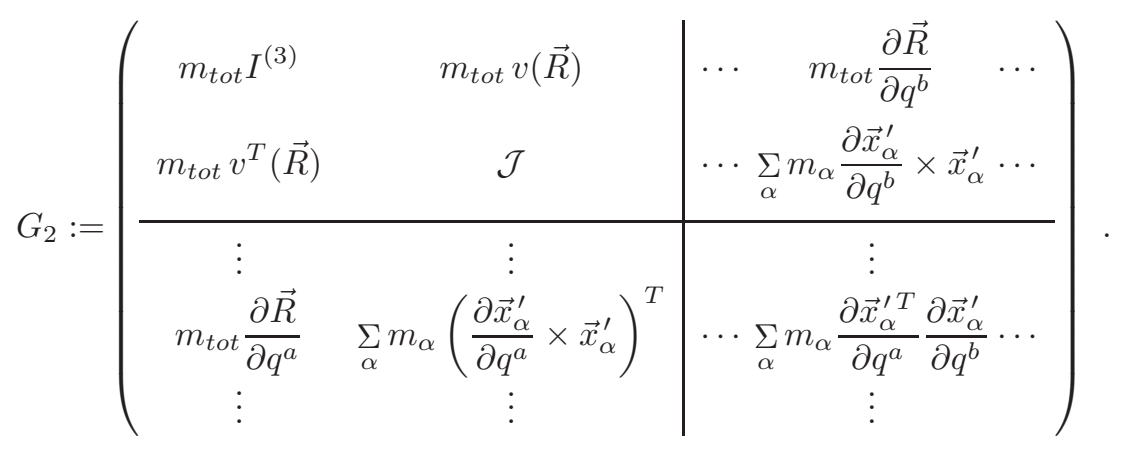

However, in this section we would like to benefit from the special structure of eq. (4.2), where, differently from the constrained case, only $N \times N$ matrices occur, and find an expression simpler than eq. (4.4).

If we take determinants on both sides of eq. (4.2), we obtain

$$
\operatorname{det} G=\left(\prod_{\alpha=1}^{n} m_{\alpha}^{3}\right) \operatorname{det}^{2} J
$$

Similarly to eq. (3.4), $J$ may be written as follows:

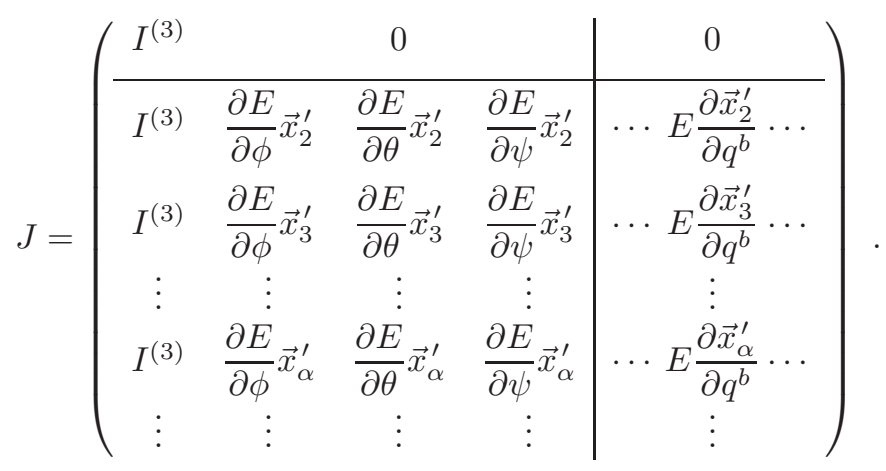

Now, the following identity is useful:

$$
J=\left(\begin{array}{cccc}
I^{(3)} & & & 0 \\
& E & & \\
& & \ddots & \\
0 & & & E
\end{array}\right) J_{1},
$$

where we have defined

$$
J_{1}:=\left(\begin{array}{cccc|c}
I^{(3)} & \multicolumn{3}{c|}{0} & 0 \\
\hline I^{(3)} & E^{T} \frac{\partial E}{\partial \phi} \vec{x}_{2}^{\prime} & E^{T} \frac{\partial E}{\partial \theta} \vec{x}_{2}^{\prime} & E^{T} \frac{\partial E}{\partial \psi} \vec{x}_{2}^{\prime} & \cdots \frac{\partial \vec{x}_{2}^{\prime}}{\partial q^{b}} \cdots \\
I^{(3)} & E^{T} \frac{\partial E}{\partial \phi} \vec{x}_{3}^{\prime} & E^{T} \frac{\partial E}{\partial \theta} \vec{x}_{3}^{\prime} & E^{T} \frac{\partial E}{\partial \psi} \vec{x}_{3}^{\prime} & \cdots \frac{\partial \vec{x}_{3}^{\prime}}{\partial q^{b}} \cdots \\
\vdots & \vdots & \vdots & \vdots & \vdots \\
I^{(3)} & E^{T} \frac{\partial E}{\partial \phi} \vec{x}_{\alpha}^{\prime} & E^{T} \frac{\partial E}{\partial \theta} \vec{x}_{\alpha}^{\prime} & E^{T} \frac{\partial E}{\partial \psi} \vec{x}_{\alpha}^{\prime} & \cdots \frac{\partial \vec{x}_{\alpha}^{\prime}}{\partial q^{b}} \cdots \\
\vdots & \vdots & \vdots & \vdots & \vdots
\end{array}\right)
$$


and we have that only the determinant of $J_{1}$ needs to be computed, since $\operatorname{det} J=$ $\operatorname{det}^{n-1} E \operatorname{det} J_{1}=\operatorname{det} J_{1}$.

Next, we note that, according to the definition of the primed reference frame in sec. 2 . some of the components of the vectors $\vec{x}_{2}^{\prime}$ and $\vec{x}_{3}^{\prime}$ are zero, namely, we have that

$$
\vec{x}_{2}^{\prime}=\left(\begin{array}{c}
0 \\
0 \\
x_{2}^{\prime 3}
\end{array}\right) \quad \text { and } \quad \vec{x}_{3}^{\prime}=\left(\begin{array}{c}
x_{3}^{\prime 1} \\
0 \\
x_{3}^{\prime 3}
\end{array}\right) \text {. }
$$

Hence, the derivatives with respect to $q^{b}$ of the zero components are also zero, rendering three zero rows in the bottom right block of eq. (4.9). Performing two row permutations so that the zero rows are the top-most ones, we obtain a matrix $J_{2}$ whose determinant is the same as the one of $J_{1}$ :

$$
J_{2}=\left(\begin{array}{ccc}
I^{(3)} & & 0 \\
& J_{2}^{\mathcal{E}} & \\
\mathcal{X} & & J_{2}^{\mathcal{I}}
\end{array}\right)
$$

where the blocks in the diagonal have been defined as

$$
J_{2}^{\mathcal{E}}=\left(\begin{array}{ccc}
\left(E^{T} \frac{\partial E}{\partial \phi} \vec{x}_{2}^{\prime}\right)^{1} & \left(E^{T} \frac{\partial E}{\partial \theta} \vec{x}_{2}^{\prime}\right)^{1} & \left(E^{T} \frac{\partial E}{\partial \psi} \vec{x}_{2}^{\prime}\right)^{1} \\
\left(E^{T} \frac{\partial E}{\partial \phi} \vec{x}_{2}^{\prime}\right)^{2} & \left(E^{T} \frac{\partial E}{\partial \theta} \vec{x}_{2}^{\prime}\right)^{2} & \left(E^{T} \frac{\partial E}{\partial \psi} \vec{x}_{2}^{\prime}\right)^{2} \\
\left(E^{T} \frac{\partial E}{\partial \phi} \vec{x}_{3}^{\prime}\right)^{2} & \left(E^{T} \frac{\partial E}{\partial \theta} \vec{x}_{3}^{\prime}\right)^{2} & \left(E^{T} \frac{\partial E}{\partial \psi} \vec{x}_{3}^{\prime}\right)^{2}
\end{array}\right),
$$

the superindices standing for vector components, and

$$
J_{2}^{\mathcal{I}}=\left(\begin{array}{ccc}
\cdots & \frac{\partial x_{2}^{\prime 3}}{\partial q^{b}} & \cdots \\
\cdots & \frac{\partial x_{3}^{\prime 1}}{\partial q^{b}} & \cdots \\
\cdots & \frac{\partial x_{3}^{\prime 3}}{\partial q^{b}} & \cdots \\
\cdots & \frac{\partial \vec{x}_{4}^{\prime}}{\partial q^{b}} & \cdots \\
& \vdots & \\
\cdots & \frac{\partial \vec{x}_{\alpha}^{\prime}}{\partial q^{b}} & \cdots \\
& \vdots &
\end{array}\right)
$$

The concrete form of the submatrix $\mathcal{X}$ in eq. (4.11) is irrelevant for our purposes, since

$$
\operatorname{det} J=\operatorname{det} J_{2}=\operatorname{det} J_{2}^{\mathcal{E}} \operatorname{det} J_{2}^{\mathcal{I}} \text {. }
$$

An explicit computation of $J_{2}^{\mathcal{E}}$ yields

$$
J_{2}^{\mathcal{E}}=\left(\begin{array}{ccc}
x_{2}^{\prime 3} \sin \theta \sin \psi & -x_{2}^{\prime 3} \cos \psi & 0 \\
x_{2}^{\prime 3} \sin \theta \cos \psi & x_{2}^{\prime 3} \sin \psi & 0 \\
-x_{3}^{\prime 1} \cos \theta+x_{3}^{\prime 3} \sin \theta \cos \psi & x_{3}^{\prime 3} \sin \psi & x_{3}^{\prime 1}
\end{array}\right)
$$

with determinant $\operatorname{det} J_{2}^{\mathcal{E}}=\sin \theta x_{3}^{\prime 1}\left(x_{2}^{\prime 3}\right)^{2}$. 
Using eqs. (4.14) and (4.6), we finally obtain

$$
\operatorname{det} G\left(q^{A}, q^{a}\right)=\sin ^{2} \theta\left(x_{3}^{\prime 1}\left(q^{a}\right)\right)^{2}\left(x_{2}^{\prime 3}\left(q^{a}\right)\right)^{4} \operatorname{det} J_{2}^{\mathcal{I}}\left(q^{a}\right)\left(\prod_{\alpha=1}^{n} m_{\alpha}^{3}\right),
$$

where the factorization has been achieved, since the only factor that depends on the external coordinates is $\sin ^{2} \theta$.

\section{Conclusions}

In this work, we have calculated explicit expressions in which the determinant of the massmetric tensor $G$ (eqs. (4.16) and (4.4) ) and the determinant of the reduced mass-metric tensor $g$ (eq. (3.19)), occurring in Classical Statistical Mechanics in the coordinate space, are written as a product of two functions; one depending only on the external coordinates that describe the overall translation and rotation of the system, and the other only on the internal coordinates. This has been done for any molecule, general internal coordinates and arbitrary constraints, extending the work in refs. 30 and 47.

This factorization allows to integrate out the external coordinates and perform Monte Carlo simulations in the internal conformational space, gaining insight of the problem, simplicity in the description of the system and, for small molecules, some computational effort. Also, our results indicate that, in general, the Fixman's compensating potential [31-34], customarily used to reproduce the stiff equilibrium distribution using rigid molecular dynamics simulations, does not depend on the external variables.

In appendix A, we give a general mathematical argument showing that the factorization is a consequence of the symmetries of the metric tensors involved and, in appendix B, the determinant of the mass-metric tensor $G$ is computed explicitly in the SASMIC [24] set of curvilinear coordinates for general branched molecules (see eq. A.10) showing that the classical formula for serial polymers [30] is actually valid for any macromolecule.

All the expressions derived in the present work are directly applicable to real cases, as has been checked in ref. 31 .

\section{Acknowledgments}

We would like to thank J. L. Alonso and F. Falceto for illuminating discussions.

This work has been supported by the Aragón Government ("Biocomputación y Física de Sistemas Complejos" group) and by the research grants MEC (Spain) FIS2004-05073 and FPA2003-02948. P. Echenique and I. Calvo are supported by MEC (Spain) FPU grants.

\section{Appendix A:}

\section{General mathematical argument}

Let $\mathcal{M}$ be a finite dimensional differentiable manifold equipped with a riemannian metric tensor. Take local coordinates $q^{\mu}$ on $\mathcal{M}$ and denote by $G_{\mu \nu}(q)$ the components of the metric tensor in these coordinates.

The transformation

$$
q^{\prime \mu}=q^{\mu}+\epsilon \xi^{\mu}(q)+O\left(\epsilon^{2}\right)
$$

is said an isometry and $\xi^{\mu}(x)$ is said a Killing vector field if 


$$
G_{\mu \nu}\left(q^{\prime}(q)\right)=J_{\mu}^{\rho}\left(q^{\prime}(q)\right) G_{\rho \sigma}(q) J_{\nu}^{\sigma}\left(q^{\prime}(q)\right)
$$

where

$$
J_{\nu}^{\mu}\left(q^{\prime}(q)\right):=\left(\frac{\partial q^{\mu}}{\partial q^{\prime \nu}}\right)\left(q^{\prime}(q)\right) .
$$

Expanding eq. A.2 up to first order in $\epsilon$ and noticing that $\operatorname{det}\left(J_{\nu}^{\mu}\right)=1-\epsilon \partial_{\mu} \xi^{\mu}(q)$, we obtain the following differential equation for $\mathcal{G}:=\operatorname{det}\left(G_{\mu \nu}\right)$ :

$$
\xi^{\mu}(q) \partial_{\mu} \mathcal{G}(q)=-2\left(\partial_{\mu} \xi^{\mu}(q)\right) \mathcal{G}(q) .
$$

Let us apply this machinery to the case considered in this work. For concreteness, we shall derive the factorization of the external coordinates in the unconstrained case and shall argue that this still holds in the constrained one.

Simultaneous translations and rotations of all the particle 2 are isometries of the massmatrix tensor in eq. (3.3). The important point for us is that, in the coordinates $q^{\mu}$ introduced in sec. 2 these transformations change the external coordinates $(X, Y, Z, \phi, \theta, \psi)$ and leave the internal coordinates $q^{a}$ untouched (see eq. (2.2)).

A global translation is given in Cartesian coordinates by $x_{\alpha}^{p} \mapsto x_{\alpha}^{p}+\epsilon$. In the coordinates $q^{\mu}$, it takes $(X, Y, Z) \mapsto(X, Y, Z)+\epsilon(1,1,1)$ and does not affect the remaining coordinates. With the above notation, $\xi^{\mu}=1, \mu=1,2,3$ and $\xi^{\mu}=0, \forall \mu>3$. Hence, eq. A.4 implies that

$$
\partial_{X} \mathcal{G}=\partial_{Y} \mathcal{G}=\partial_{Z} \mathcal{G}=0
$$

i.e., the determinant of the mass-metric tensor does not depend on the coordinates $X, Y, Z$.

A global rotation in the coordinates $q^{\mu}$ rotates $(X, Y, Z)$ and changes the Euler angles (in a complicated way which will not be important for our purposes) but does not affect the internal coordinates. Hence, $\xi^{\mu}=0, \forall \mu>6$. In addition, the matrix $J_{\nu}^{\mu}$ does not depend on $X, Y, Z$ because the rotation acts linearly on them. Let us abbreviate $\alpha \equiv \alpha^{p} \equiv(\phi, \theta, \psi), p=1, \ldots, 3$. Recalling that $\mathcal{G}$ does not depend on $X, Y, Z$, the differential equation (A.4) reads

$$
\xi^{p}(\alpha) \partial_{p} \mathcal{G}\left(\alpha, q^{a}\right)=-2\left(\partial_{p} \xi^{p}(\alpha)\right) \mathcal{G}\left(\alpha, q^{a}\right) .
$$

The group of rotations in $\mathbb{R}^{3}$ has three linearly independent Killing vector fields which are complete in the sense that one can join two arbitrary points $(\phi, \theta, \psi)$ and $\left(\phi^{\prime}, \theta^{\prime}, \psi^{\prime}\right)$ by moving along integral curves of the Killing vector fields. This guarantees that the solution of eq. (A.6) is of the form

$$
\mathcal{G}\left(\alpha, q^{a}\right)=\mathcal{G}_{1}(\alpha) \mathcal{G}_{2}\left(q^{a}\right)
$$

and we have the desired result.

To derive the factorization of the external coordinates in the constrained case, simply notice that the constraints in this work do not involve the external coordinates. Therefore, global translations and rotations are still isometries of the reduced mass-metric tensor and the result follows.

\footnotetext{
${ }^{2}$ Notice that the isometry group of the mass-metric tensor is much bigger, since translations and rotations acting independently on each particle are also isometry transformations.
} 


\section{Appendix B:}

\section{Determinant of $\mathrm{G}$ in particular coordinates}

The SASMIC scheme, introduced in ref. 24, is a set of rules to define particular Zmatrix coordinates $[59,60]$ of general branched molecules, with convenient properties of modularity and approximate separability of soft and hard modes.

According to the rules, to each atom $\alpha$, one uniquely assigns three atoms $\beta(\alpha), \gamma(\alpha)$ and $\delta(\alpha)$ in such a way that the three Z-matrix internal coordinates that position atom $\alpha$ are

$$
\begin{aligned}
& r_{\alpha}:=(\alpha, \beta(\alpha)) \\
& \theta_{\alpha}:=(\alpha, \beta(\alpha), \gamma(\alpha)) \\
& \phi_{\alpha}:=(\alpha, \beta(\alpha), \gamma(\alpha), \delta(\alpha)),
\end{aligned}
$$

being $r_{\alpha}$ a bond length, $\theta_{\alpha}$ a bond angle and $\phi_{\alpha}$ a dihedral angle.
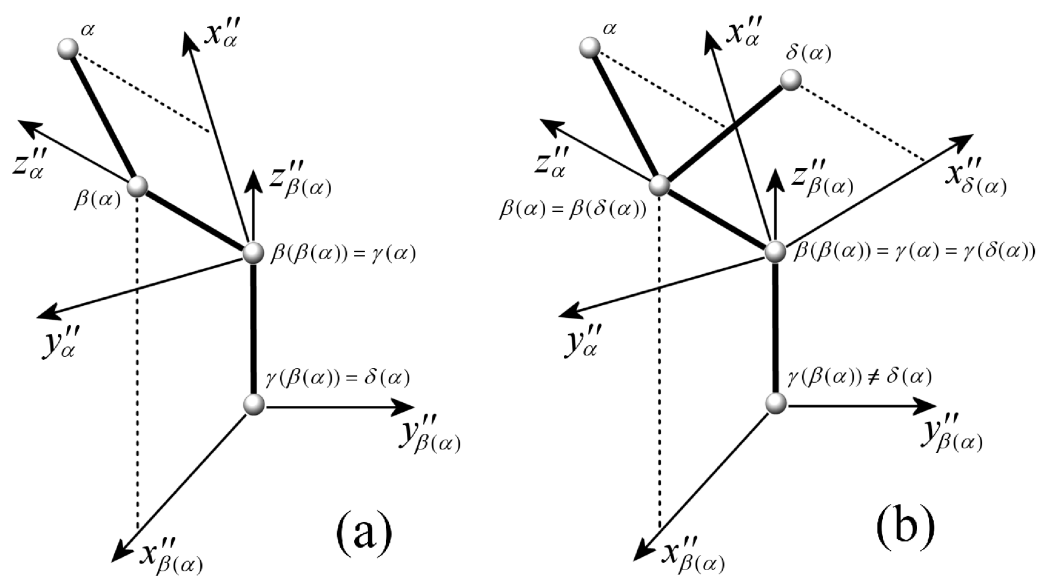

Figure 2: Local reference frames associated to atoms $\alpha$ and $\beta(\alpha)$ (see text) in the cases that (a) $\phi_{\alpha}$ is a principal dihedral or (b) $\phi_{\alpha}$ is a phase dihedral.

The procedure that will be followed in order to express the position $\vec{x}_{\alpha}^{\prime}$ of atom $\alpha$ in the primed reference frame in fig. 1 as a function of the SASMIC internal coordinates starts by expressing the vector that goes from $\beta(\alpha)$ to $\alpha$ in a set of axes $\left(x_{\alpha}^{\prime \prime}, y_{\alpha}^{\prime \prime}, z_{\alpha}^{\prime \prime}\right)$ associated to $\alpha$. This local reference frame is defined such that the $z_{\alpha}^{\prime \prime}$-axis lies along the directional bond $(\gamma(\alpha), \beta(\alpha))$ and the $x_{\alpha}^{\prime \prime}$-axis lies along the projection of $(\beta(\alpha), \alpha)$ onto the plane orthogonal to $(\gamma(\alpha), \beta(\alpha))$ (see fig. 2).

In these axes, the components of the vector $(\beta(\alpha), \alpha)$ are

$$
\vec{x}_{\alpha}^{\prime \prime T}:=\left(r_{\alpha} \sin \theta_{\alpha}, 0,-r_{\alpha} \cos \theta_{\alpha}\right) .
$$

Now, if the atom $\delta(\alpha)$ that is used to define $\phi_{\alpha}$ is bonded to atom $\gamma(\alpha)$ (fig. 2 2 a), $\phi_{\alpha}$ is called a principal dihedral [24] and we have that

$$
\underbrace{\left(\begin{array}{ccc}
-\cos \theta_{\beta(\alpha)} & 0 & \sin \theta_{\beta(\alpha)} \\
0 & 1 & 0 \\
-\sin \theta_{\beta(\alpha)} & 0 & -\cos \theta_{\beta(\alpha)}
\end{array}\right)}_{\Theta\left(\theta_{\beta(\alpha)}\right)} \underbrace{\left(\begin{array}{ccc}
\cos \phi_{\alpha} & -\sin \phi_{\alpha} & 0 \\
\sin \phi_{\alpha} & \cos \phi_{\alpha} & 0 \\
0 & 0 & 1
\end{array}\right)}_{\Phi\left(\phi_{\alpha}\right)} \vec{x}_{\alpha}^{\prime \prime}
$$


are the components of the vector $(\beta(\alpha), \alpha)$ in the local reference frame $\left(x_{\beta(\alpha)}^{\prime \prime}, y_{\beta(\alpha)}^{\prime \prime}, z_{\beta(\alpha)}^{\prime \prime}\right)$ associated to atom $\beta(\alpha)$.

On the other hand, if we are at a branching point and the atom $\delta(\alpha)$ that is used to define $\phi_{\alpha}$ is bonded to atom $\beta(\alpha)$ (fig. 20), $\phi_{\alpha}$ is called a phase dihedral [24] and we have to change first to the local reference frame associated to $\delta(\alpha)$. In this case, the components of the vector $(\beta(\alpha), \alpha)$ in the local reference frame $\left(x_{\beta(\alpha)}^{\prime \prime}, y_{\beta(\alpha)}^{\prime \prime}, z_{\beta(\alpha)}^{\prime \prime}\right)$ are

$$
\Theta\left(\theta_{\beta(\alpha)}\right) \Phi\left(\phi_{\delta(\alpha)}\right) \Phi\left(\phi_{\alpha}\right) \vec{x}_{\alpha}^{\prime \prime} .
$$

If we iterate the procedure, by changing the axes to the ones associated to the atom $\beta(\beta(\alpha))$, i.e., the $\beta$ atom that correspond to $\beta(\alpha)$ according to the SASMIC scheme, an so on, we will eventually arrive to the set of axis $\left(x_{3}^{\prime \prime}, y_{3}^{\prime \prime}, z_{3}^{\prime \prime}\right)$ (since, in the SASMIC scheme [24], we have that $\beta(\alpha)<\alpha$ ). Note however that, according to the definition of the local reference frame given in the preceding paragraphs, the one associated to atom 3 is exactly the primed reference frame in fig. 1.

Hence, let us define, for each atom $\alpha$, a matrix $R_{\alpha}$ as the product of the matrices obtained using eqs. A.3 and (A.4) and successively applying the function $\beta(\alpha)$. Then, $R_{\alpha}$ takes the vector $(\beta(\alpha), \alpha)$ in eq. (A.2) to the primed reference frame.

Let the superindex on $\beta$ denote composition of functions, let us define $\beta^{0}(\alpha):=\alpha$ and $\mathcal{N}_{\alpha}$ as the integer such that $\beta^{\mathcal{N}_{\alpha}+1}(\alpha)=3$. Adding all the vectors corresponding to $\left(\beta^{p+1}(\alpha), \beta^{p}(\alpha)\right)$ in the primed reference frame, with $p=0, \ldots, \mathcal{N}_{\alpha}$, to $\vec{x}_{3}^{\prime}$ yields the position of atom $\alpha$ in the primed reference frame as a function of the internal coordinates:

$$
\vec{x}_{\alpha}^{\prime}=\vec{x}_{3}^{\prime}+\sum_{p=\mathcal{N}_{\alpha}}^{0} R_{\beta^{p}(\alpha)} \vec{x}_{\beta^{p}(\alpha)}^{\prime \prime} .
$$

Now, ordering the internal coordinates as $\left(r_{2}, r_{3}, \theta_{3}, r_{4}, \theta_{4}, \phi_{4}, \ldots, r_{n}, \theta_{n}, \phi_{n}\right)$ and using the already mentioned fact that $\beta(\alpha)<\alpha$, and also that $\delta(\alpha)<\alpha$, we have that the matrix $J_{2}^{\mathcal{I}}$ in eq. (4.13) is

$$
J_{2}^{\mathcal{I}}=\left(\begin{array}{cccc}
A_{0} & & & 0 \\
& A_{4} & & \\
& & \ddots & \\
\mathcal{X} & & & A_{n}
\end{array}\right) \quad \text { and } \quad \operatorname{det} J_{2}^{\mathcal{I}}=\operatorname{det} A_{0} \prod_{\alpha=4}^{n} \operatorname{det} A_{\alpha} .
$$

Using that

$$
\vec{x}_{2}^{\prime}=\left(\begin{array}{c}
0 \\
0 \\
r_{2}
\end{array}\right) \quad \text { and } \quad \vec{x}_{3}^{\prime}=\left(\begin{array}{c}
r_{3} \sin \theta_{3} \\
0 \\
r_{2}-r_{3} \cos \theta_{3}
\end{array}\right)
$$

we have

$$
A_{0}:=\left(\begin{array}{ccc}
\frac{\partial x_{2}^{\prime 3}}{\partial r_{2}} & \frac{\partial x_{2}^{\prime 3}}{\partial r_{3}} & \frac{\partial x_{2}^{\prime 3}}{\partial \theta_{3}} \\
\frac{\partial x_{3}^{\prime 1}}{\partial r_{2}} & \frac{\partial x_{3}^{\prime 1}}{\partial r_{3}} & \frac{\partial x_{3}^{\prime 1}}{\partial \theta_{3}} \\
\frac{\partial x_{3}^{\prime 3}}{\partial r_{2}} & \frac{\partial x_{3}^{\prime 3}}{\partial r_{3}} & \frac{\partial x_{3}^{\prime 3}}{\partial \theta_{3}}
\end{array}\right)=\left(\begin{array}{ccc}
1 & 0 & 0 \\
0 & \sin \theta_{3} & r_{3} \cos \theta_{3} \\
1 & -\cos \theta_{3} & r_{3} \sin \theta_{3}
\end{array}\right) .
$$

Now, we note that the matrix $\Phi\left(\phi_{\alpha}\right)$ occurs always at the right-most place in $R_{\alpha}$ and that the derivatives in the blocks $A_{\alpha}$, with $\alpha>4$, kill all the terms in eq. A.5 except for the one corresponding to $p=0$. Hence, if we define $R_{\alpha}=: R_{\alpha}^{\prime} \Phi\left(\phi_{\alpha}\right)$, the block $A_{\alpha}$ may be expressed as follows: 


$$
\begin{aligned}
& A_{\alpha}:=R_{\alpha}^{\prime}\left(\frac{\partial \Phi\left(\phi_{\alpha}\right) \vec{x}_{\alpha}^{\prime \prime}}{\partial r_{\alpha}} \frac{\partial \Phi\left(\phi_{\alpha}\right) \vec{x}_{\alpha}^{\prime \prime}}{\partial \theta_{\alpha}} \frac{\partial \Phi\left(\phi_{\alpha}\right) \vec{x}_{\alpha}^{\prime \prime}}{\partial \phi_{\alpha}}\right)= \\
& \left(\begin{array}{ccc}
\sin \theta_{\alpha} \cos \phi_{\alpha} & r_{\alpha} \cos \theta_{\alpha} \cos \phi_{\alpha} & -r_{\alpha} \sin \theta_{\alpha} \sin \phi_{\alpha} \\
\sin \theta_{\alpha} \sin \phi_{\alpha} & r_{\alpha} \cos \theta_{\alpha} \sin \phi_{\alpha} & r_{\alpha} \sin \theta_{\alpha} \cos \phi_{\alpha} \\
-\cos \theta_{\alpha} & r_{\alpha} \sin \theta_{\alpha} & 0
\end{array}\right) \text {. }
\end{aligned}
$$

Finally, using eq. (A.6), noting that $\operatorname{det} A_{0}=r_{3}$ and $\operatorname{det} A_{\alpha}=-r_{\alpha}^{2} \sin \theta_{\alpha}$, and calculating the remaining terms of eq. (4.16) with eq. (A.7), we obtain the desired result:

$$
\operatorname{det}^{\frac{1}{2}} G\left(q^{A}, q^{a}\right)=\left(\prod_{\alpha=1}^{n} m_{\alpha}^{3 / 2}\right)|\sin \theta|\left(\prod_{\alpha=2}^{n} r_{\alpha}^{2}\right)\left(\prod_{\alpha=3}^{n}\left|\sin \theta_{\alpha}\right|\right) .
$$

It is worth remarking at this point that the previous expression does not explicitly depend on the dihedral angles $\phi_{\alpha}$ and that it is the same result as the one found in ref. 30 for serial polymers.

\section{References}

[1] S. B. Chen. Monte Carlo simulations of conformations of chain molecules in a cylindrical pore. J. Chem. Phys., 123:074702, 2005.

[2] D. Shental-Benchor, S. Kirca, N. Ben-Tal, and T. Haliloglu. Monte Carlo studies of folding, dynamics and stability in $\alpha$-helices. Biophys. J., 88:2391-2402, 2005.

[3] J. Klos and T. Pakula. Lattice Monte Carlo simulations of three-dimensional charged polymer chains. J. Chem. Phys., 120:2496-2501, 2005.

[4] L. Nivón and E. I. Shakhnovich. All-atom monte Carlo simulation of GCAA RNA folding. J. Mol. Biol., 344:29-45, 2004.

[5] J. Shimada and E. I. Shakhnovich. The ensemble folding kinetics of protein G from an all-atom monte Carlo simulation. Proc. Natl. Acad. Sci. USA, 99:11175-11180, 2002.

[6] U. H. E. Hansmann and Y. Okamoto. New Monte Carlo algorithms for protein folding. Curr. Opin. Struct. Biol., 9:177-183, 1999.

[7] H. Senderowitz and W. C. Still. Sampling potential energy surface of glycyl glycine peptide: Comparison of Metropolis Monte Carlo and stochastic dynamics. J. Comp. Chem., 19:1294-1299, 1998.

[8] N. G. Almarza, E. Enciso, J. Alonso, F. J. Bermejo, and M. Álvarez. Monte Carlo simulations of liquid n-butane. Mol. Phys., 70:485-504, 1990.

[9] J. L. Alonso, G. A. Chass, I. G. Csizmadia, P. Echenique, and A. Tarancón. Do theoretical physicists care about the protein folding problem? In R. F. ÁlvarezEstrada et al., editors, Meeting on Fundamental Physics 'Alberto Galindo'. Aula Documental, Madrid, 2004. (arXiv:q-bio.BM/0407024).

[10] C. M. Dobson. Protein folding and misfolding. Nature, 426:884-890, 2003.

[11] S. S. Plotkin and J. N. Onuchich. Structural and energetic heterogeneity in protein folding. I. Theory. J. Chem. Phys., 116:5263-5283, 2000.

[12] K. A. Dill. Polymer principles and protein folding. Prot. Sci., 8:1166-1180, 1999. 
[13] R. D. Taylor, P. J. Jewsbury, and J. W. Essex. A review of protein-small molecule docking methods. J. Comput. Aid. Mol. Des., 16:151-166, 2002.

[14] G. R. Smith and M. J. E. Sternberg. Prediction of protein-protein interactions by docking methods. Curr. Opin. Struct. Biol., 12:28-35, 2002.

[15] M. Levantino, Q. Huang, A. Cupane, M. Laberge, A. Hagarman, and R. SchweitzerStenner. The importance of vibronic perturbations in ferrocytochrome c spectra: A reevaluation of spectral properties based on low-temperature optical absortion, resonance raman, and molecular-dynamics simulations. J. Chem. Phys., 123:054508, 2000 .

[16] L. D. Barron and Hecht L. Vibrational raman optical activity: from fundamentals to biochemical applications. In K. Nakanishi, N. Berova, and R. W. Woody, editors, Circular Dichroism Principles and Applications, pages 667-701. Wiley-VCH, New York, 2nd edition, 2000.

[17] S. Yang and M. Cho. IR spectra of N-methylacetamide in water predicted by combined quantum mechanical/molecular mechanical molecular dynamics simulations. J. Chem. Phys., 123:134503, 2005.

[18] H. H. Mantsch and Chapman D. Infrared Spectroscopy of Biomolecules. Wiley-Liss, Chichester, UK, 1996.

[19] N. Sreerama and R. W. Woody. Circular dichroism of peptides and proteins. In K. Nakanishi, N. Berova, and R. W. Woody, editors, Circular Dichroism Principles and Applications, pages 601-620. Wiley-VCH, New York, 2nd edition, 2000.

[20] J.-H. Choi, J.-S. Kim, and M. Cho. IR spectra of N-methylacetamide in water predicted by combined quantum mechanical/molecular mechanical molecular dynamics simulations. J. Chem. Phys., 122:174903, 2005.

[21] T. A. Keiderling. Peptide and protein conformational studies with vibrational circular dichroism and related spectroscopies. In K. Nakanishi, N. Berova, and R. W. Woody, editors, Circular Dichroism Principles and Applications, pages 621-666. Wiley-VCH, New York, 2nd edition, 2000.

[22] J. T. Pelton and L. R. McLean. Spectroscopic methods for analysis of protein secondary structure. Anal. Biochem., 277:167-176, 200.

[23] D. A. Case, H. J. Dyson, and P. E. Wright. Use of chemical shifts and coupling constants in nuclear magnetic resonance structural studies on peptides and proteins. Methods Enzymol., 239:392-416, 1994.

[24] P. Echenique and J. L. Alonso. Definition of Systematic, Approximately Separable and Modular Internal Coordinates (SASMIC) for macromolecular simulation. To be published in J. Comp. Chem., 2006. (arXiv:q-bio.BM/0511004).

[25] C. J. Cramer. Essentials of Computational Chemistry: Theories and Models. John Wiley \& Sons, Chichester, 2nd edition, 2002.

[26] G. A. Chass, M. A. Sahai, J. M. S. Law, S. Lovas, Ö. Farkas, A. Perczel, J.-L. Rivail, and I. G. Csizmadia. Toward a computed peptide structure database: The role of a universal atomic numbering system of amino acids in peptides and internal hierarchy of database. Intl. J. Quant. Chem., 90:933-968, 2002. 
[27] R. A. Abagyan, M. M. Totrov, and D. A. Kuznetsov. ICM: A new method for protein modeling and design: Applications to docking and structure prediction from the distorted native conformation. J. Comp. Chem., 15:488-506, 1994.

[28] A. K. Mazur and R. A. Abagyan. New methodology for computer-aided modelling of biomolecular structure and dynamics. 1. Non-cyclic structures. J. Biomol. Struct. Dyn., 6:815-832, 1989.

[29] R. A. Abagyan and A. K. Mazur. New methodology for computer-aided modelling of biomolecular structure and dynamics. 2. Local deformations and cycles. J. Biomol. Struct. Dyn., 6:833-845, 1989.

[30] N. Gō and H. A. Scheraga. On the use of classical statistical mechanics in the treatment of polymer chain conformation. Macromolecules, 9:535, 1976.

[31] P. Echenique, I. Calvo, and J. L. Alonso. Quantum mechanical calculation of the effects of stiff and rigid constraints in the conformational equilibrium of the alanine dipeptide. Submitted to J. Comp. Chem, 2006. (arXiv:q-bio.QM/0601042).

[32] D. C. Morse. Theory of constrained Brownian motion. Adv. Chem. Phys., 128:65$189,2004$.

[33] W. K. Den Otter and W. J. Briels. Free energy from molecular dynamics with multiple constraints. Mol. Phys., 98:773-781, 2000.

[34] M. Fixman. Classical Statistical Mechanics of constraints: A theorem and application to polymers. Proc. Natl. Acad. Sci. USA, 71:3050-3053, 1974.

[35] D. Perchak, J. Skolnick, and R. Yaris. Dynamics of rigid and flexible constraints for polymers. Effect of the Fixman potential. Macromolecules, 18:519-525, 1985.

[36] M. Pasquali and D. C. Morse. An efficient algorithm for metric correction forces in simulations of linear polymers with constrained bond lengths. J. Chem. Phys., 116:1834, 2002.

[37] H. M. Chun, C. E. Padilla, D. N. Chin, M. Watanabe, V. I. Karlov, H. E. Alper, K. Soosaar, K. B. Blair, O. M. Becker, L. S. D. Caves, R. Nagle, D. N. Haney, and B. L. Farmer. MBO(N)D: A multibody method for long-time Molecular Dynamics simulations. J. Comp. Chem., 21:159-184, 2000.

[38] S. Reich. Smoothed Langevin dynamics of highly oscillatory systems. Physica D, 118:210-224, 2000.

[39] J. Zhou, S. Reich, and B. R. Brooks. Elastic molecular dynamics with self-consistent flexible constraints. J. Chem. Phys., 111:7919, 2000.

[40] S. He and H. A. Scheraga. Brownian dynamics simulations of protein folding. J. Chem. Phys., 108:287, 1998.

[41] H. J. C. Berendsen and W. F. Van Gunsteren. Molecular Dynamics simulations: Techniques and approaches. In A. J. et al. Barnes, editor, Molecular LiquidsDynamics and Interactions, pages 475-500. Reidel Publishing Company, 1984.

[42] M. R. Pear and J. H. Weiner. Brownian dynamics study of a polymer chain of linked rigid bodies. J. Chem. Phys., 71:212, 1979. 
[43] D. Chandler and B. J. Berne. Comment on the role of constraints on the conformational structure of n-butane in liquid solvent. J. Chem. Phys., 71:5386-5387, 1979.

[44] E. Helfand. Flexible vs. rigid constraints in Statistical Mechanics. J. Chem. Phys., 71:5000, 1979 .

[45] M. Fixman. Simulation of polymer dynamics. I. General theory. J. Chem. Phys., $69: 1527,1978$

[46] J. M. Rallison. The role of rigidity constraints in the rheology of dilute polymer solutions. J. Fluid Mech., 93:251-279, 1979.

[47] A. Patriciu, G. S. Chirikjian, and R. V. Pappu. Analysis of the conformational dependence of mass-metric tensor determinants in serial polymers with constraints. J. Chem. Phys., 121:12708-12720, 2004.

[48] J. Chen, W. Im, and C. L. Brooks III. Application of torsion angle molecular dynamics for efficient sampling of protein conformations. J. Comp. Chem., 26:1565-1578, 2005 .

[49] B. Hess, H. Saint-Martin, and H. J. C. Berendsen. Flexible constraints: An adiabatic treatment of quantum degrees of freedom, with application to the flexible and polarizable mobile charge densities in harmonic oscillators model for water. J. Chem. Phys., 116:9602, 2002.

[50] H. J. C. Berendsen and W. F. Van Gunsteren. Molecular Dynamics with constraints. In J. W. Perram, editor, The Physics of Superionic Conductors and Electrode Materials, volume NATO ASI Series B92, pages 221-240. Plenum Press, 1983.

[51] W. F. Van Gunsteren and M. Karplus. Effects of constraints on the dynamics of macromolecules. Macromolecules, 15:1528-1544, 1982.

[52] N. Gō and H. A. Scheraga. Analysis of the contributions of internal vibrations to the statistical weights of equilibrium conformations of macromolecules. J. Chem. Phys., 51:4751, 1969.

[53] E. B. Wilson Jr., J. C. Decius, and P. C. Cross. Molecular Vibrations: The Theory of Infrared and Raman Vibrational Spectra. Dover Publications, New York, 1980.

[54] A. Láng, I. G. Csizmadia, and A. Perczel. Peptide models. XLV: Conformational properties of N-formyl-L-methioninamide ant its relevance to methionine in proteins. PROTEINS: Struct. Funct. Bioinf., 58:571-588, 2005.

[55] A. Perczel, O. Farkas, I. Jakli, I. A. Topol, and I. G. Csizmadia. Peptide models. XXXIII. Extrapolation of low-level Hartree-Fock data of peptide conformation to large basis set SCF, MP2, DFT and $\operatorname{CCSD}(\mathrm{T})$ results. The Ramachandran surface of alanine dipeptide computed at various levels of theory. J. Comp. Chem., 24:1026$1042,2003$.

[56] R. Vargas, J. Garza, B. P. Hay, and D. A. Dixon. Conformational study of the alanine dipeptide at the MP2 and DFT levels. J. Phys. Chem. A, 106:3213-3218, 2002 .

[57] C.-H. Yu, M. A. Norman, L. Schäfer, M. Ramek, A. Peeters, and C. van Alsenoy. $\mathrm{Ab}$ initio conformational analysis of N-formyl L-alanine amide including electron correlation. J. Mol. Struct., 567-568:361-374, 2001. 
[58] A. G. Császár and A. Perczel. Ab initio characterization of building units in peptides and proteins. Prog. Biophys. Mol. Biol., 71:243-309, 1999.

[59] W. J. Hehre, W. A. Lathan, R. Ditchfield, M. D. Newton, and J. A. Pople. Gaussian 70. Quantum Chemistry Program Exchange, 1970. Program No. 237.

[60] I. N. Levine. Quantum Chemistry. Prentice Hall, Upper Saddle River, 5th edition, 1999. 